\title{
The Neutrophil to Lymphocyte Ratio: An Ideal Marker for Early Diagnosis and Short-Term Prognosis of Acute Kidney Injury?
}

\author{
Susanne M. Lang ${ }^{\mathrm{a}}$ Helmut Schiffl ${ }^{\mathrm{b}}$ \\ aKlinik für Innere Medizin II, University Hospital FSU Jena, Jena, Germany; ${ }^{b}$ Department of Internal Medicine IV, \\ University Hospital, Munich, Germany
}

Hospital-acquired acute kidney injury (AKI) is a common heterogeneous renal syndrome. It is characterized by multiple causes and risk factors, a broad spectrum of clinical manifestations ranging from tubular damage to kidney failure, and unpredictable outcomes. The earlier an injurious process to the kidneys can be identified, the timelier preventive and supportive measures (removal of the precipitating factor for injury and renal replacement therapy [RRT]) can be initiated. There is a pressing unmet clinical need for novel biomarkers with improved diagnostic and prognostic ability [1].

Mediators of inflammation play an important role in the pathogenesis of ischemic, nephrotoxic, or septic AKI. Dr. Zhu and colleagues [2] explored the ability of the neutrophil to lymphocyte ratio (NLR) as defined by the quotient of the absolute neutrophil and lymphocyte counts, to predict adverse outcomes (need for RRT, mortality) of hospitalized patients with AKI. The main results of their retrospective analyses support the basic concept that the marker NLR has diagnostic and prognostic potential in inflammation-mediated diseases [2]. However, at the

karger@karger.com www.karger.com/kdd

Karger"

BOPEN ACCESS
(C) 2021 The Author(s)

Published by S. Karger AG, Basel

This is an Open Access article licensed under the Creative Commons Attribution-NonCommercial-4.0 International License (CC BY-NC) (http://www.karger.com/Services/OpenAccessLicense), applicable to the online version of the article only. Usage and distribution for commercial purposes requires written permission. current stage, there are still too many unresolved concerns about the validity and reliability of the results and uncertainties regarding the mechanisms that underly the association between an elevated NLR and poor prognosis.

First, no prospective randomized trial tested the predictive ability of NLR in patients with AKI. The limitations of all retrospective cohort studies include the lack of central blinded adjudication of clinical events, incomplete assessment of known confounders (cigarette smoking, obesity, and comorbid diseases), and inconsistent documentation of prescribed or self-administered medications. Retrospective studies cannot distinguish between causes and events.

Second, cross-sectional study analyses capture a patient population at a single point in time. The timing of the snapshot may differ among illnesses with defined or unknown onset of AKI (post-cardiac surgery AKI, contrast media-induced nephropathy, and combined renal insults). Most novel biomarkers of AKI demonstrate a dynamic pattern reflective of the cellular events that occur at different time points throughout the clinical phases of 
AKI. Moreover, not all patients diagnosed with AKI stage 1 progress to stage 2 or 3 (need of RRT). Thus, multiple measurements at different time points are necessary rather than a single test to predict the need for initiation of RRT.

Third, different values of NLR, measured with different methods in different AKI populations, are cited in the literature. No single value is currently defined for patients at risk for AKI, progression of AKI, or mortality from AKI. All studies chose arbitrary thresholds based either on the median or the higher quartile of NLR, or they used cut-off values determined by receiver-operating curves to predict the occurrence, progression, or mortality of AKI [2-6]. Both the sensitivity and specificity of calculated cutoffs were modest, and none of the cutoffs was prospectively and externally validated [2-4]. There is a wide variation in the thresholds for high NLR among the studies ranging from 2.6 [4] to 17.1 [7]. The cut-off values for NLR were highest in septic patients treated in the intensive care unit, intermediate in patients with post-cardiac surgery, and lowest (within the normal reference range) in patients undergoing coronary angiography or transcatheter aortic valve replacement. However, there was a wide range of cutoffs within specific subgroups of AKI patients and a significant overlap of NLR values between patients and healthy subjects [3]. NLR is not a specific biomarker of kidney damage or kidney function. It is a marker of the acuity of the illness associated with systemic inflammation. The assumption that high NLRs are prognostic for all AKI patients regardless of demographic factors or clinical characteristics is most likely incorrect. Retrospective stratification of AKI patients by specific cutoffs is a further source of selection bias.

Forth, no cutoff for NLR in AKI patients has been proposed based on reference values coming from healthy populations. There is a need for these reference values to discriminate normal from abnormal results. The normal NLR values in an adult, nongeriatric US population without any acute illness and/or chronic debilitating disease were between 0.78 and 3.53 with a mean NLR of 2.13. None of the NLRs assessed in the validation series was outside of the proposed limits [8].

Higher NLRs were noted in older, male, or postmenopausal female Caucasians or obese normal subjects $[8,9]$. Well-conducted retrospective analyses of the association of NLR and diagnosis, progression, or mortality of AKI revealed significant differences in patient characteristics, comorbidities, and biochemical parameters between survivors and nonsurvivors $[2,5]$. There were also differences in cardiovascular surgery patients with or without
AKI [5]. In the retrospective study by Zhu et al. [2], nonsurvivors had a higher mean NLR of 6.8 (ranges 3.3-13.2) compared to survivors with a NLR of 4.4 (ranges 2.5-9.3). Nonsurvivors were older, had more comorbidities (diabetes, cardiovascular diseases; chronic liver injury, and COPD), and higher AKI stages requiring more often RRT, as well as lower albumin and hematocrit values. The retrospective analysis by Kim et al. [5] showed that postcardiac surgery patient with AKI or without AKI differed significantly in median NLR (2.3 vs. 1.98 ) but also in the portion of elderly patients, in the number of heavy smokers or patients with preexisting chronic renal diseases as well as in relevant surgical details (duration of cardiopulmonary bypass time and blood loss). Patient groups differed not only in the markers of inflammation but also in major patient-associated risk factors and treatment-related precipitating factors of AKI. It may prove impossible to find retrospectively subgroups of patients for NLR studies that have remarkably similar demographic characteristics, clinical features, and treatment regimens except for 1 variable (AKI).

Fifth, whether NLR can predict AKI earlier than the traditional biomarker $\mathrm{Cr}$ or urine output remains unknown, the results of the studies in various clinical settings are inconsistent. The meta-analyses of Chen et al. [3] indicated that the NLR may be a reliable marker for the early detection of AKI. In contrast, Zhu et al. [2] reported that high NLR showed a predictive power comparable to serum Cr. Regrettably, none of the studies tested simultaneously other biomarkers for AKI.

Sixth, less research has been done to compare NLR with established markers of inflammation or infection, so we do not know whether NLR in combination with other biomarkers can improve its clinical utility: Seventh, the literature is full of studies using NLR in patients with cancer, pulmonary diseases, cardiovascular diseases, liver diseases, schizophrenia, hearing loss, and more. Overall, the utility of NLR remains unclear. However, if NLR is an indicator of every disease, then it is not an indicator of a specific disease. NLR obviously cannot be a magical assay for every condition.

Finally, neither earlier diagnosis of AKI nor earlier prediction of requirement of RRT translates into earlier treatment and better patient outcome. To date, there are no interventions beyond treatment of the underlying disease and good medical care. No pharmacologic drug has been proven to reverse established AKI or slow down the progression of AKI. Earlier initiation of RRT was not associated with improved mortality of severe AKI. By contrast, there is concern that misleading or false positive 
NLR may prevent or delay angiographic procedures or the administration of potentially nephrotoxic antibiotics.

We acknowledge that studies testing the diagnostic and prognostic ability of higher NLRs have shown very impressive results and agree that the validated biomarker has the potential to transform the way we diagnose and treat patients with AKI. However, there are steep barriers on the road to clinical application of NLR in patients with AKI. Future studies should identify and validate clinically meaningful thresholds in high-risk patient subgroups and determine whether clinical implementation of this biomarker is a realistic and attainable goal.

\section{Conflict of Interest Statement}

The authors declare no conflicts of interest.

\section{Funding Sources}

There was no funding.

\section{Author Contributions}

The authors contributed substantially to the conception of the commentary.

\section{References}

1 Schiffl H, Lang SM. Update on biomarkers of acute kidney injury: moving closer to clinical impact? Mol Diagn Ther. 2012;16(4):199207.

2 Zhu J, Zeng C, Zhang L, Shu S, Liu Y, Chen G, et al. Red blood cell distribution width and neutrophil-to-lymphocyte ratio in predicting adverse outcomes of acute kidney injury in hospitalized patients. Kidney Dis. 2020;6(5): 371-81.

3 Chen D, Xiao D, Guo J, Chahan B, Wang Z. Neutrophil-lymphocyte count ratio as a diagnostic marker for acute kidney injury: a systematic review and meta-analysis. Clin Exp Nephrol. 2020;24(2):126-35.
4 Yelgec NS, Karatas MB, Karabay CY, Canga Y, Calik AN, Simsek B, et al. The relationship between acute renal failure after transcatheter aortic valve replacement and preprocedural neutrophil to lymphocyte ratio. Dicle Med. 2020;47:1-9.

5 Kim WH, Park JY, Ok SH, Shin IW, Sohn JT. Association between the neutrophil/lymphocyte ratio and acute kidney injury after cardiovascular surgery: a retrospective observational study. Medicine. 2015;94(43):e1867.

6 Canakci E, Karatas A, Kaya Y. Can blood groups, neutrophil-lymphocyte ratio and mean platelet volume predict mortality in critically ill patients developing acute kidney injury. J Anesth Critical Care. 2017;8:00290.
$7 \mathrm{Bu} \mathrm{X}$, Zhang L, Chen P, Wu X. Relation of neutrophil-to-lymphocyte ratio to acute kidney injury in patients with sepsis and septic shock: a retrospective study. Int Immunopharmacol. 2019;70:372-7.

8 Forget P, Khalifa C, Defour JP, Latinne D, Van Pel MC, De Kock M. What is the normal value of the neutrophil-to-lymphocyte ratio? BMC Res Notes. 2017;10(1):12.

9 Li J, Chen Q, Luo X, Hong J, Pan K, Lin X, et al. Neutrophil-to-lymphocyte ratio positively correlates to age in healthy population. J Clin Lab Anal. 2015;29(6):437-43. 